\title{
Are bacteria the main pathological factor in pathogenesis of lower limb varicose veins and non-healing ulcers?
}

\author{
WL Olszewski ${ }^{1 \dagger}$, MT Zaleska ${ }^{1,2}$, E Stelmach $^{3}$ \\ ${ }^{1}$ Depart.of Vascular Surgery, Central Clinical Hospital, Ministry of Internal Affairs \\ 2 Dept.of Applied Physiology, Mossakowski Medical Research Center, Polish Academy of Sciences \\ 3 Dept. of Clinical Microbiology, Warsaw Medical University
}

submitted: Jan 14, 2020, accepted: Dec 5, 2020, EPub Ahead of Print: Dec 14, 2020

Conflict of interest: None

DOI: 10.24019/jtavr.91 - Corresponding author:Dr. Marzanna T Zaleska, mzaleska34@gmail.com

(C) 2019 Fondazione Vasculab impresa sociale ONLUS. All rights reserved.

\begin{abstract}
Why do the saphenous vein varices arise? According to our concept this is a process of slow deconstruction of the vein wall by inflammation caused by microbes penetrating plantar skin, colonizing deep tissues, entering veins and sticking to valves, as well as, transported to lymphatics, bringing about damage to their wall and evoking immune response in the inguinal lymph nodes. Venous stasis caused by a variety of hemodynamic factors remains secondary to the primary damage of the vein wall. With respect to ulcer, irrespective of the predisposing factors as venous stasis, colonization of the denuded calf surfaces takes place by bacteria drained from foot skin and also those floating down to calf from the perineum.
\end{abstract}

\section{Introduction}

Why do the saphenous vein varices arise? What is the primary factor responsible for destructive changes in the vein wall leading to loss of mechanical resistance to the flowing blood force? The commonly accepted notion is the effect of venous blood pressure exerted upon the wall. Hydrostatic pressure, destructed valves, low effectiveness of leg muscle contractions are upon the list. However, what is the primary factor weakening the wall resistance to pressure, decreasing its thickness and increasing vein radius? Our concept is a process of slow deconstruction of the vein wall by inflammation caused by microbes penetrating plantar skin, colonizing deep tissues, entering veins and sticking to valves, as well as, flowing to lymphatics, bringing about damage to their wall and evoking immune response in the inguinal
This set of microbes is responsible for progression of ulcer or its delayed healing. In this study, the numerical phenotypes of varicose veins and ulcer granulation tissue bacteria were presented. The dominant strains in the vein walls were Staph. epidermidis and aureus with Enterococci and Pseudomonas frequent in ulcers. High percentage of Staphylococci both in vein walls and ulcer sensitive to antibiotics may suggest that they originated from the microbiome inhabiting leg tissues prior to the development of varices and ulcer.

Keywords Venous ulcers, Cryptic bacteria, Persister cells, Infection, Skin

lymph nodes (Fig. 1). Venous stasis due to a variety of hemodynamic factors is secondary to the primary damage of the vein walls. Nevertheless, the role of hemodynamics in the development of tissue changes caused by retrograde capillary hypertension remains crucial and adds to the pathological picture e.g. by formation of edema and ulcer.

What is a lower leg ulcer? It is a circumscribed necrosis of epidermis, skin, and occasionally also muscular fascia, tendon or even underlying bone with sluggish granulation and delayed covering by keratinocytes migrating from the ulcer margins (Fig. 2). The inflammatory reaction is comprising a several times larger mass of tissue than the ulcer itself (Fig. 3-4). Following skin micro-injury the colonization of denuded surface by local skin and floating down perineal bacterial flora takes place. 
Factors predisposing to
decreased venous return Right heart insufficiency Obesity with pelvic vein flow functional obstruction

\author{
Deep vein insufficiency \\ Valve destruction, wall stiffness
}

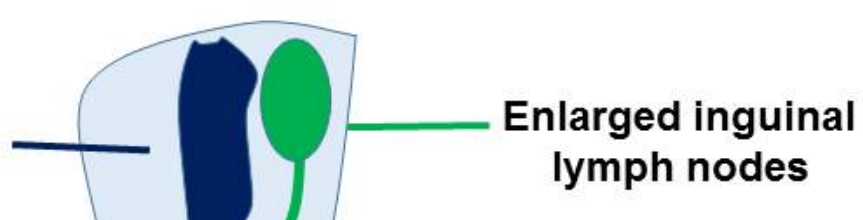

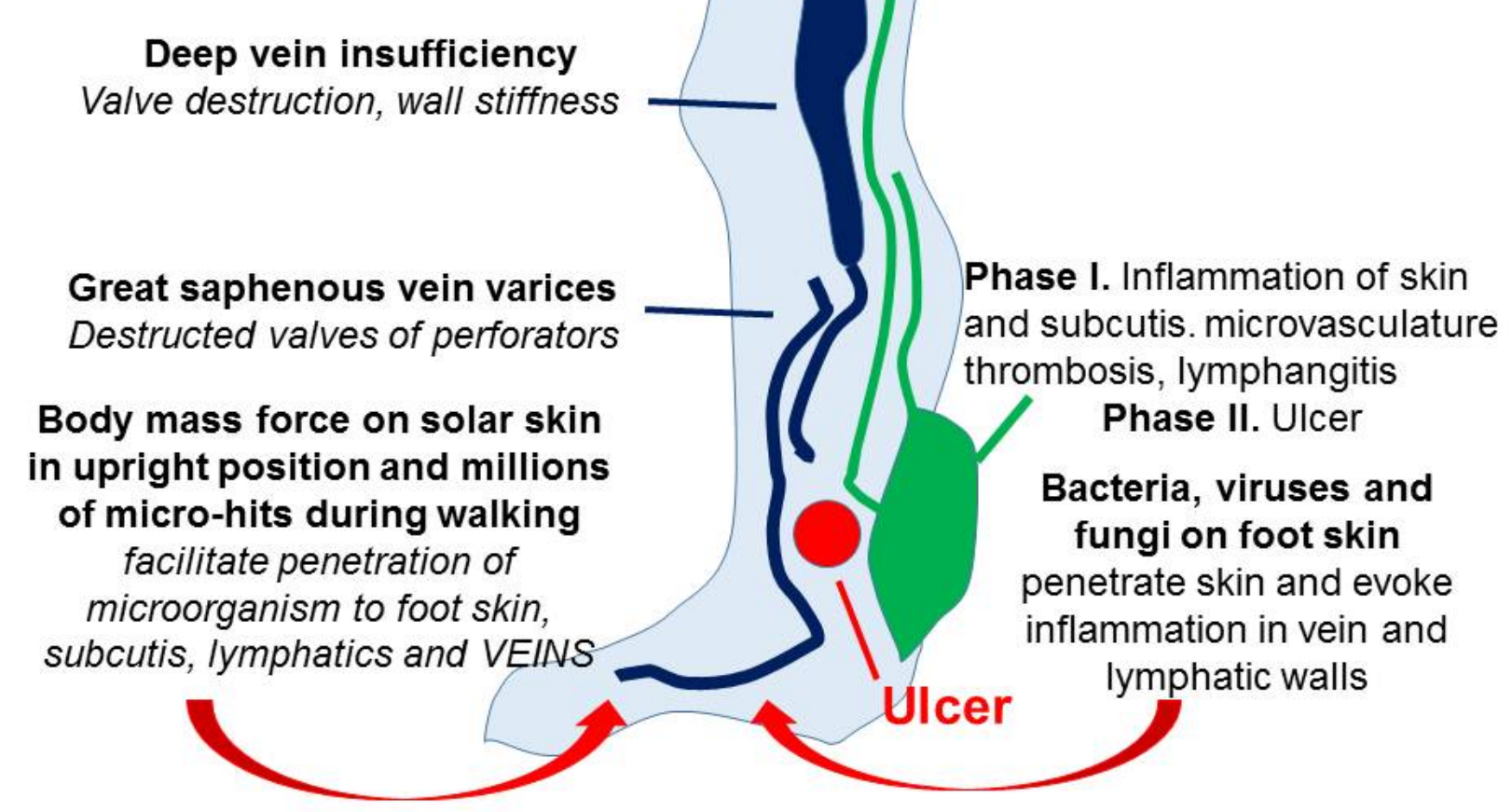

Fig. 1 - Schematic presentation of the lymphatic system participating in elimination of microbes penetrating foot skin in normal conditions (right leg on drawing and lymphoscintigram) and in left leg with calf ulcer (red dot and extravasation of isotope on lymphoscintigram). Arrow shows sites of penetration and transport lymphatic pathways of microbes, reaction of inguinal and retroperitoneal nodes, flow to the thoracic dust and subclavian blood.

The predisposing factors are venous stasis with excess capillary filtrate, high tissue fluid pressure, erythrocyte extravasation, hemosiderosis and fibrosis, or ischemia in atherosclerosis and diabetes with decreased arterial supply of nutrients, or lymph stasis with excess tissue fluid, high tissue fluid pressure and fibrosis, or excessive fat deposition in the subcutis in pathological obesity with excess tissue fluid and high tissue fluid pressure. The common denominator, irrespective of the predisposing factors, is colonization of the veins and denuded calf skin surface by bacteria drained from foot and floating down to calf from the perineum.

\section{Lower limb skin bacteria}

Human skin has been considered to harbor a complex microbial ecosystem, with transient, short-term resident and long-term resident biota, based on the consistency with which they are isolated. Staphylococcus, Micrococcus,
Corynebacterium, Brevibacteria, Propionibacteria, and Acinetobacter species are, among others, regularly cultivated from normal skin. Staphylococcus aureus, Streptococcus pyogenes, and Pseudomonas aeruginosa may be transient colonizers, especially in pathological conditions $^{1,2}$. These strains colonize any denuded skin surface of lower limbs, among them ulcers.

\section{Could bacteria be present in and around the varicose veins?}

The exact etiology of varicose vein formation and development of ulcer is still full of questions. Are these two entities linked with each other? Could dormant persister bacteria in leg subcutaneous tissue be responsible for vein wall destruction and subsequently ulcer formation?

We tried to detect bacteria in the varicose veins (CEAP 2) and subcutis using two techniques. 

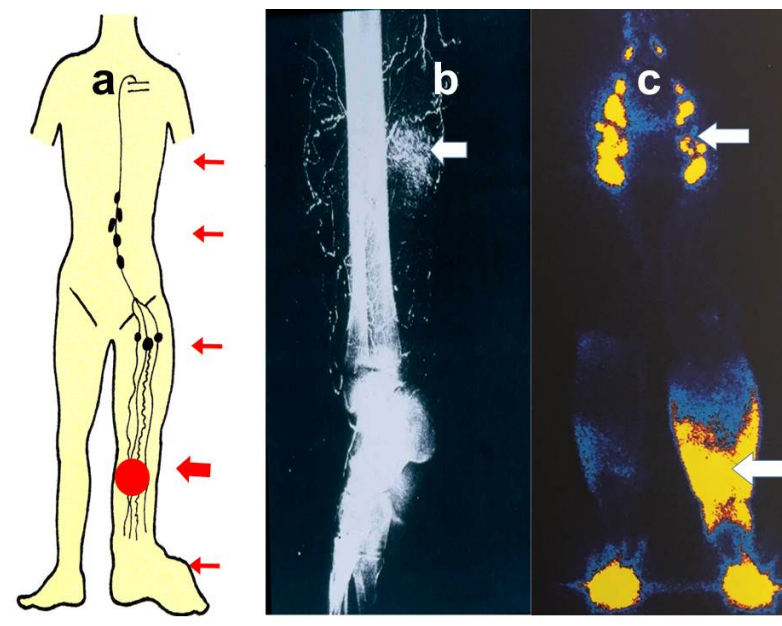

Fig. 2 - a. Classic X-ray Lipiodol lymphogram showing extravasation of marker in the bottom of ulcer and a dense network of fragmented lymphatics. B. Lymphoscintigram of lower limb with calf ulcer (lower arrow) and decreasing in size fibrotic lymph nodes (upper arrow). Lymph nodes and vessels were damaged in course of spread of inflammation from calf ulcer.
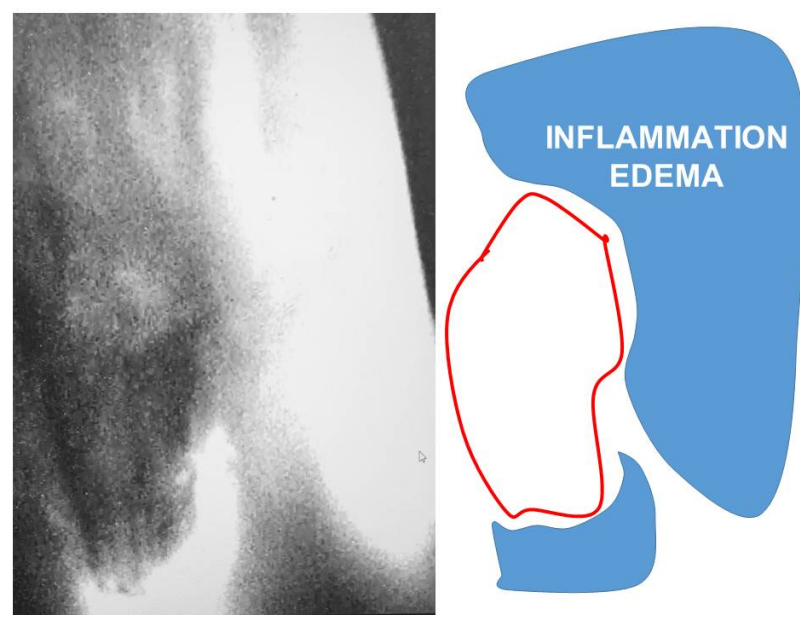

Fig. 3 - Indocyanine green fluorescent lymphography after injection into the foot lymphatics extravasating around the ulcer (red circle) showing how large area of calf tissues is involved in inflammation.

Biopsy material was homogenized and cultured in routine media and in another method, it was placed on bacteriological culture plates and observed for 3 weeks. In this last technique, tissue environment for bacteria was preserved and plate contained erythrocytes (iron). This mimicked for bacteria a normal in-tissue



Fig. 4 - a. Indocyanine green lymphogram shows area of necrosis and around it extravasation of the dye in the surrounding inflamed area. $b$. infra-red thermogram depicting the extended area of increased temperature in the inflamed region around the ulcer. Inflammation process region is definitely larger than that of ulcer itself.



Fig. 5 - Fragments of the saphenous vein (left arrow) and adjacent subcutaneous tissue (right arrow) on Hemoline bacteriological culture plate with con-fluent bacterial colonies (arrows). Bacteria grew supplied by tissue nutrients and "crawled" upon culture plate. Days or weeks of culture in warm-box were needed in most cases to see bacterial proliferation.

situation. In addition, bacterial 16sRNA was identified in specimens ${ }^{3}$. The control studies to eliminate the possibility of contamination included instruments, gauze and gloves. Operating room bacterial $6 \mathrm{~h}$ fall-out was done routinely with sporadically 1-2 Staphylococci colonies per plate. 


\begin{tabular}{|lccc}
\hline Type & Specimens & Infected & $\%$ \\
GSV (varicose) & 100 & 40 & 40 \\
GSV + femoral vein (normal) & 25 & 4 & 16 \\
Skin above varix (after disinfection) & 100 & 4 & 4
\end{tabular}

Table I - Bacteriology of varices of the great saphenous veins (GSV).

\begin{tabular}{lcc}
\hline & Varicose GSV & $\begin{array}{c}\text { Control GSV } \\
\text { and femoral vein }\end{array}$ \\
Staphylococci: coagulase-neg & 22 & \\
- epidermidis & 13 & \\
- hominis & 4 & \\
- hemolyticus & 2 & 1 \\
- capitis & 1 & \\
- warneri & 1 & \\
- intermedius & 1 & 1 \\
S.aureus & 10 & \\
Micrococcus spp & 2 & 1 \\
Branhamella catarhalis & 1 & \\
Aerococcus viridans & 1 & \\
Enterococcus faecium & 3 & \\
Acinetobacter & 1 & \\
Gemella morbil & 1 & \\
Strept. mitis & 1 & \\
Pseudomonas & & \\
\end{tabular}

Table II - Bacterial isolates from varicose great saphenous vein (GSV).

\section{Bacterial isolates in varicose veins}

In our studies, varicose veins specimens stage 4 (CEAP classification) revealed presence of bacterial isolates in $40 \%$, whereas controls taken from healthy cadaveric organ donors contained live bacteria in only 4\%.(Table I and II) Disinfected skin specimens from the sites of varicectomy showed presence of microbes in $4 \%$. The dominant isolates from vein specimens were Staphylococci, preponderantly coagulase negative, however, in a few cases Enterococcus faecium was also detected. Staphylococci were highly sensitive to antibiotics except of penicillin (Table III). Thirty-three percent of isolates were methicillin-resistant. The 16sRNA was detected in $69 \%$ of specimens, evidently higher than the percentage of live bacterial cells ${ }^{3}$.
Bacterial culture on the Hemoline plates revealed microbes migrating from the outer aspect of varices, adjacent fat but not muscles (Fig.5)

\section{Bacterial isolates on ulcers}

Bacterial phenotypes on ulcer exudate remained similar to those identified on adjacent skin, however, the numerical distribution of strains became different. Strains of gram-ve Bacilli dominated over others and the number of colonies was doubled (Table IV ). This may be the results of more favorable environmental conditions on the granulation tissue for some strains or less favorable for others. Interestingly, bacteria cultured from the ulcer surface revealed increasing resistance to antibiotics compared with the flora taken from the normal leg skin (Table V) ${ }^{3}$. Still, sensitivity to antibiotics remained high. 


\begin{tabular}{lcc}
\hline & Staph.coagulase-negative & Staph. aureus \\
Penicillin & 32 & 27 \\
Cotrimoxazole & 95 & 91 \\
Gentamycin & 95 & 82 \\
Erythromycin & 68 & 63 \\
Clindamycin & 74 & 73 \\
Tetracyclines & 68 & 73 \\
Minocycline & 100 & 100 \\
Vancomycin & 100 & 100 \\
Teicoplanin & 100 & 100 \\
Rifampicin & 100 & 100 \\
Nor/Quinolones & 74 & 91 \\
Fusidic acid & 79 & 91 \\
Nitrofurantoin & 84 & 91 \\
Quinupristine & 100 & 100 \\
Oxacillin & 73 & 67
\end{tabular}

Table III - Sensitivity to antibiotics of bacterial isolates from varicose fragments of the great saphenous vein. $n=40$ specimens (in \%).






\begin{tabular}{|c|c|c|c|c|c|}
\hline \multirow[t]{3}{*}{ Antibiotic } & \multicolumn{5}{|c|}{ Cocci } \\
\hline & \multicolumn{2}{|c|}{ Varicose ulcer } & & \multicolumn{2}{|c|}{ Normals } \\
\hline & +++ & + & & +++ & + \\
\hline Penicillin & $31 * *$ & 12 & & 28 & 2 \\
\hline Oxacillin & 55 & 0 & $*$ & 73 & 0 \\
\hline Kanamycin & 25 & 8 & $*$ & 44 & 8 \\
\hline Tobramycin & 29 & 35 & $*$ & 75 & 15 \\
\hline Gentamycin & 48 & 22 & $*$ & 85 & 4 \\
\hline Tetracyclin & 28 & 42 & & 61 & 2 \\
\hline Minocyclin & 16 & 16 & $*$ & 100 & 0 \\
\hline Erythromycin & 43 & 13 & & 59 & 8 \\
\hline Lincomycin & 37 & 12 & $*$ & 69 & 6 \\
\hline Pristinamycin & 70 & 20 & & 100 & 0 \\
\hline Fosfomycin & 45 & 8 & & 77 & 0 \\
\hline Rifampicin & 77 & 14 & & 91 & 9 \\
\hline Fusidic acid & 63 & 27 & & 77 & 18 \\
\hline Vancomycin & 58 & 8 & $*$ & 88 & 2 \\
\hline Cotrimoxazole & 46 & 15 & $*$ & 63 & 16 \\
\hline
\end{tabular}

Table V - Sensitivity to antibiotics of bacterial isolates from 18 leg varicose ulcers and calf skin of 30 normal controls. $* p<0.05 ; * * \%$ of sensitive isolates.

\begin{tabular}{|lcccccc}
\hline Site of isolation & Strains/patient & \multicolumn{2}{c}{ Cocci } & \multicolumn{2}{c}{ Bacilli } \\
& & Gram +ve & Gram -ve & $\begin{array}{c}\text { spore } \\
\text { forming } \\
\text { spore non-forming }\end{array}$ \\
Perineum & 2.40 & 66.7 & 0 & 0 & 13.9 & 19.4 \\
Calf Skin & 1.47 & 68.2 & 4.5 & 9.1 & 4.5 & 13.7 \\
Toeweb & 2.16 & 84.6 & 0 & 0 & 0 & 15.4 \\
Venous ulcer & $4.84^{*}$ & $38.9 *$ & 0 & 0 & 7.9 & $53.2 *$
\end{tabular}

Table VI - Frequency of bacteria isolates on perineal skin, calf skin, toeweb and varicose ulcer (in \%). p<0.05.

Out of 17 toeweb isolates, 13 were of the same phenotype as perineal isolates (Table VI). The isolates were less sensitive to antibiotics than controls from skin. Interestingly, around $40 \%$ of ulcer bacteria were Staphylococci.

Bacteria beside of being present on skin surface and in skin appendices, may dwell in soft tissues in a dormant persister state ${ }^{5}$. They can be activated for proliferation upon stimuli as trauma, inflammation, ulcer, etc.
There are still several question to be answered before we can achieve a progress in healing of ulcers. They are:

- $\quad$ 1. Why does ulcer occur more frequently in the calf and foot than in the upper limbs or elsewhere?

- 2. Is it local micro-trauma that initiates ulcer development?

- 3. Why does a rapid colonization of ulcer by skin residential bacteria develop so fast ?

- 4. Do perineal bacteria contribute to the ulcer colonization? 
- $\quad$ 5. Do the normally saprophytic skin bacteria become virulent once they colonize the denuded surface?

6. Why do the colonizing bacteria proliferate rapidly increasing the bacteria cell mass ?

- 7. Which bacterial strains dominate

- 8. Are there dormant bacteria in calf subcutaneous tissue (persisters) ?

9. Does a local cellular memory to bacterial antigens exist stimulating immediate recruitment of granulocytes and macrophages ?

- 10. Is there autoimmune reaction to own granulocyte and tissue debris and insufficient granulocyte autophagy of incorporated bacteria ?

Taken together, presence of Staphylococci in CEAP 2 varicose GSV wall strongly suggests their causative role in damage to vein structures. It may be presumed that inflammation evoked by the Staphyloccoci antigens leads gradually to CEAP 3, 4 and 5 and eventually 6 which is an ulcer. Ulcer is colonized by Staphylococci originating from skin surface already present in varices, whereas gram-negative bacteria drifting from perineum secondarily populate the granulation tissue.

\section{References}

1) Kloos WE, Musselwhite MS. Distribution and persistence of Staphylococcus and Micrococcus species and other aerobic bacteria on human skin. Appl Microbiol. 1975 Sep;30(3):381-5. PMID: 810086; PMCID: PMC187193.

2) Costello EK, Lauber CL, Hamady M, Fierer N, Gordon JI, Knight R. Bacterial community variation in human body habitats across space and time. Science. 2009 Dec 18;326(5960):1694-7. doi: $10.1126 /$ science.1177486. Epub 2009 Nov 5. PMID: 19892944; PMCID: PMC3602444.

3) Olszewski WL, Zaleska MT, Stelmach E, Swoboda-Kopec E, Jain P, Agrawal K, GogiaS , Gogia A, Andziak P. Cryptic bacteria of lower limb deep tissues as a possible cause of inflammatory and necrotic changes in ischemia, venous stasis and varices, and lymphedema. Surg Infect (Larchmt). 2015 Jun;16(3):313-22. doi: 10.1089/sur.2014.019

4) Halbert AR, Stacey MC, Rohr JB, Jopp-McKay A. The effect of bacterial colonization on venous ulcer healing. Australas J Dermatol. 1992;33(2):75-80. doi: 10.1111/j.1440-0960.1992.tb00083.x. PMID: 1294056.

5) Lewis K. Persister cells. Annu Rev Microbiol. 2010;64:357-72. doi: 10.1146/annurev.micro.112408.134306. PMID: 20528688. 
